Received: 2017.04.25 Accepted: 2017.07.03 Published: 2017.07.25

Authors' Contribution:

Study Design A

Data Collection B

Statistical Analysis C

Data Interpretation D

Manuscript Preparation E

Literature Search F

Funds Collection $G$

Corresponding Author: Conflict of interest:

\title{
A Case Report of Cystic Pheochromocytoma
}

\author{
EF 1 Shoaib Z. Junejo \\ DEF 2 Sandeep Tuli \\ E 3 David M. Heimann \\ F 4 Issac Sachmechi \\ EF 4 David Reich
}

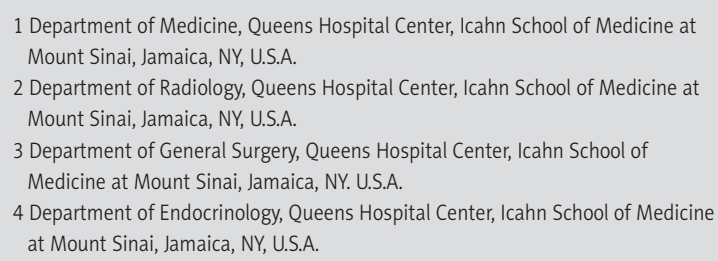

1 Department of Medicine, Queens Hospital Center, Icahn School of Medicine at Mount Sinai, Jamaica, NY, U.S.A.

2 Department of Radiology, Queens Hospital Center, Icahn School of Medicine at Mount Sinai, Jamaica, NY, U.S.A.

3 Department of General Surgery, Queens Hospital Center, Icahn School of Medicine at Mount Sinai, Jamaica, NY. U.S.A.

4 Department of Endocrinology, Queens Hospital Center, Icahn School of Medicine at Mount Sinai, Jamaica, NY, U.S.A.

\begin{abstract}
Patient: Male, 64
Final Diagnosis: Cystic pheochromocytoma

Symptoms: Abdominal pain

Medication: -

Clinical Procedure: -

Specialty: Endocrinology and Metabolic

Objective: Rare disease

Background: Pheochromocytoma is a rare catecholamine-producing tumor with an estimated incidence of less than $0.1 \%$ in the global population. We present a case of cystic pheochromocytoma that was diagnosed as an incidental finding. The patient presented with abdominal pain and had a history of hypertension.

Case Report: A 64-year-old man with hypertension presented with a clinical history of intermittent abdominal pain for one year. He denied sweating, palpitations, headache or back pain. He was found to have an elevated blood pressure of 170/90 and no palpable abdominal mass. Contrast-enhanced computed tomography (CT) imaging of the abdomen and pelvis were performed that showed cystic mass measuring $9 \mathrm{~cm}$ in diameter arising from the left adrenal gland with contrast-enhancing mural nodules. Magnetic resonance imaging (MRI) confirmed the cystic nature of the mass. Laboratory analysis showed an elevated plasma normetanephrine (NMN) of 1,087 pg/ml and metanephrine (MN) of $372 \mathrm{pg} / \mathrm{ml}$; 24-hour urine showed elevated levels of NMN and MN, 3,002 mg/24 h and $1,596 \mathrm{mg} / 24 \mathrm{~h}$, respectively. Given the laboratory and radiologic findings, a diagnosis of cystic pheochromocytoma was made. After controlling blood pressure with the alpha-blocker, doxazosin, the patient was hydrated and scheduled for an elective adrenalectomy. The histopathology of the excised adrenal gland was consistent with a cystic pheochromocytoma.

Conclusions: Cystic pheochromocytoma is a very rare tumor that may present without symptoms. The clinical course of cystic pheochromocytoma is similar to that of solid pheochromocytoma. Early surgical intervention is recommended, following blood pressure control with an alpha-blocker, and adequate hydration.
\end{abstract}

MeSH Keywords: Metanephrine $・$ Normetanephrine $\bullet$ Pheochromocytoma

Full-text PDF: http://www.amjcaserep.com/abstract/index/idArt/905042 


\section{Background}

Pheochromocytoma is a rare catecholamine-producing tumor with an estimated incidence of less than $0.1 \%$ in the global population [1]. Pheochromocytoma commonly arises from the adrenal medulla, but extra-adrenal pheochromocytoma can occur [2]. Although this tumor is usually solid, it can present as a cystic lesion [3]. Solid and cystic pheochromocytoma can be differentiated by imaging and histology $[2,3]$. Solid pheochromocytoma may show calcification on imaging, while cystic pheochromocytoma is due to hemorrhagic degeneration, necrosis, and cyst formation [2,3]. Few cases of cystic pheochromocytoma have been reported in the literature. We present a case of cystic pheochromocytoma that was discovered as an incidental finding (incidentaloma).

\section{Case Report}

A 64-year-old man with a history of hypertension presented with intermittent abdominal pain for one year. He denied sweating, palpitations, headache or back pain. On physical examination, he was found to have an elevated blood pressure of $170 / 90$ and no palpable abdominal mass. Contrast-enhanced computed tomography (CT) imaging of the abdomen and pelvis were performed and showed a cystic mass measuring approximately $9 \mathrm{~cm}$ in diameter arising from the left adrenal gland with enhancing mural nodules (Figure 1). Mural nodules occurred within the adrenal gland and were unilocular, noncalcified, hypo-attenuated masses, each with a thin wall (Figure 1). Magnetic resonance imaging (MRI) confirmed the cystic nature of the mass with hemorrhagic degeneration, necrosis, cyst formation and mural nodules (Figure 2).
Laboratory investigations showed an elevated plasma normetanephrine (NMN) of $1,087 \mathrm{pg} / \mathrm{ml}$ and metanephrine (MN) of $372 \mathrm{pg} / \mathrm{ml}$. A 24-hour urine analysis showed elevated levels of NMN and MN, 3,002 mg/24 h and 1,596 mg/24 h, respectively. Repeat plasma levels of NMN and MN were also elevated and did not change much three hours after the patient took 0.3 $\mathrm{mg}$ of clonidine. Given the laboratory and imaging findings, a provisional diagnosis of cystic pheochromocytoma was made.

After the patient's blood pressure was controlled with the alpha-blocker, doxazosin, the patient was hydrated and scheduled for an elective adrenalectomy. Following removal of the adrenal gland, histopathology confirmed a diagnosis of pheochromocytoma with cystic degeneration.

Six months following surgery, the patient remains stable with no tumor recurrence and his most recent ambulatory blood pressure was 105/69 on low-dose amlodipine and lisinopril.

\section{Discussion}

Pheochromocytoma is a tumor arising from adrenomedullary chromaffin cells, which produce and secrete catecholamines [1,2]. The triad of symptoms of headache, sweating, and palpitations in patients with hypertension is diagnostic, with a $94 \%$ specificity and $91 \%$ sensitivity; between $20 \%$ and $30 \%$ are clinically asymptomatic [3]. There are several reports in the literature describing cystic pheochromocytoma [2-4]. The main clinical presentation of pheochromocytoma is hypertension, which is seen in $90 \%$ of cases, of which, two-thirds have paroxysmal hypertensive episodes, along with tachycardia, palpitations, headaches, sweating, tremors, and anxiety $[3,5]$. Symptoms due to pheochromocytoma may also include nausea, vomiting, and chest and abdominal pain. Signs

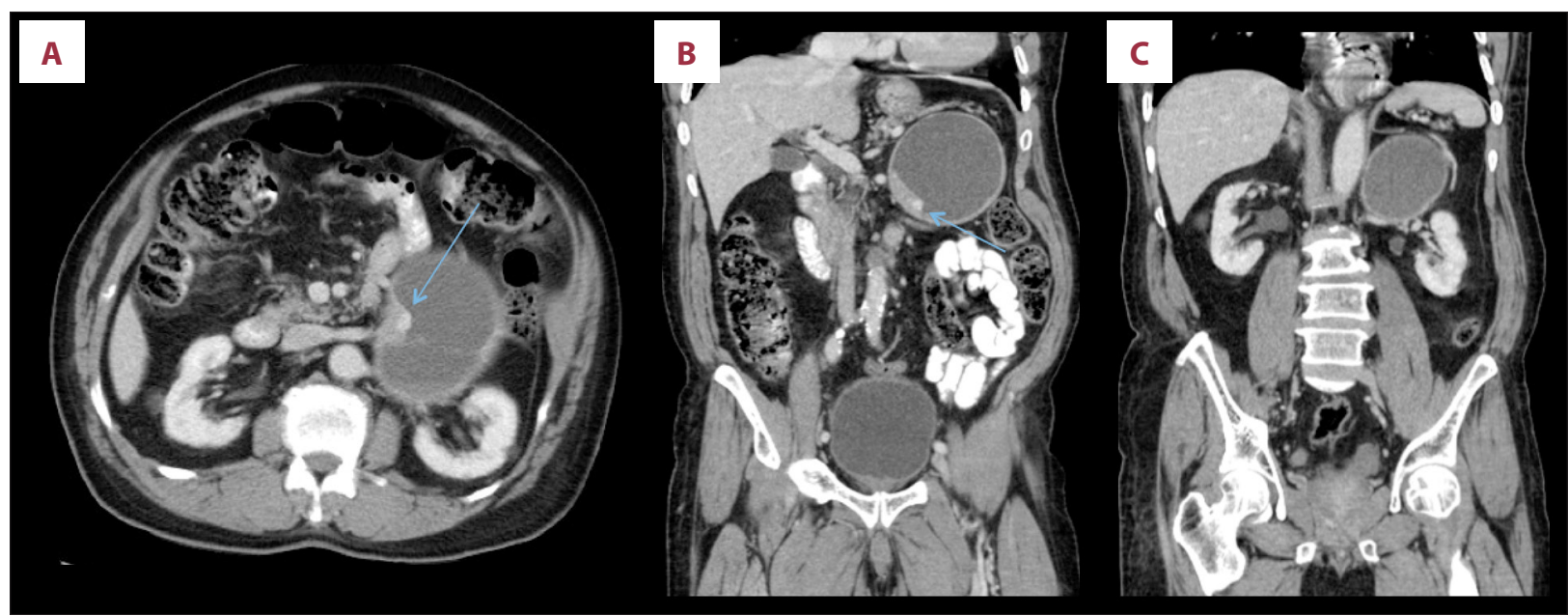

Figure 1. Axial and coronal contrast-enhanced abdominal computed tomography (CT) imaging. Axial (A) and coronal (B, C) contrastenhanced CT images show a cystic mass arising from the left adrenal that contains mural nodules with enhancement (arrow). 


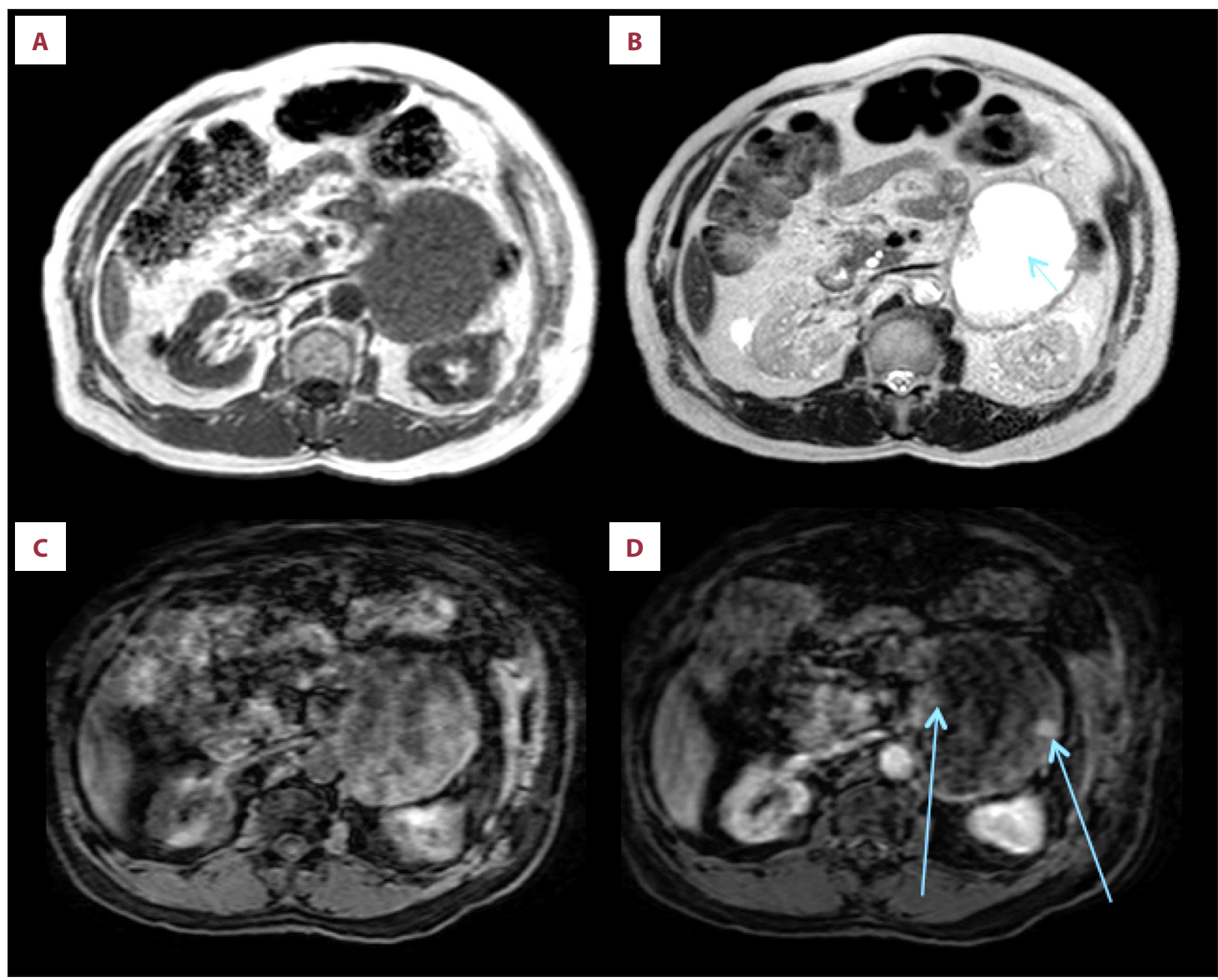

Figure 2. Axial T1-weighted and T2-weighted magnetic resonance imaging (MRI). Axial MRI images: T1 weighted (A), T2 weighted (B), precontrast (C), and postcontrast (D) images demonstrate a cystic mass in left adrenal that has layering debris (short arrow in B). Mass is isointense to muscle on T1 imaging and hyperintense on T2 imaging with hypointense nodules along the wall. On post contrast-enhanced imaging, there is enhancement in the mural nodules that is not seen on the pre-contrast image (large arrow in D).

and symptoms are often paroxysmal because of the episodic nature of catecholamine secretion by pheochromocytoma $[5,6]$.

Pheochromocytoma should be considered as a diagnosis in patients presenting with nonspecific symptoms and an incidental cystic adrenal mass [6]. It can be difficult to diagnose cystic pheochromocytoma, as on imaging they can resemble benign adrenal cysts and do not show the typical imaging features of solid pheochromocytoma, including the magnetic resonance imaging (MRI) features [6].

In this case report, the patient presented with abdominal pain associated with high blood pressure, and had a cystic lesion in the left adrenal gland, with the surgical histopathology findings confirming pheochromocytoma. Formation of a cystic pheochromocytoma may be due to hemorrhagic degeneration, necrosis cyst formation. The definitive treatment for pheochromocytoma is surgical excision and histological examination. In this case, an early diagnosis was made, and the patient underwent surgery following adequate preparation, including blood pressure control. The consequences of a delay in diagnosis and treatment of a patient with pheochromocytoma include an increased risk of morbidity and mortality due to myocardial infarction and stroke.

\section{Conclusions}

Cystic pheochromocytoma is a very rare tumor that may present without symptoms. The clinical course of cystic pheochromocytoma is similar to that of solid pheochromocytoma. Early surgical intervention is recommended, following blood pressure control with an alpha-blocker, and adequate hydration. 


\section{References:}

1. Dahia PL: Evolving concepts in pheochromocytoma and paraganglioma. Curr Opin Oncol, 2006; 18: 1-8

2. Lee TH, Slywotsky CM, Lavelle MT, Garcia RA: Cystic pheochromocytoma. Radiographics, 2002; 22: 935-40

3. Bush WH, Elder JS, Crane RE, Wales LR: Cystic pheochromocytoma. Urology, 1985; 25: 332-34
4. Klingler PJ, Fox TP, Menke DM et al: Pheochromocytoma in an incidentally discovered asymptomatic cystic adrenal mass. Mayo Clin Proc, 2000; 75: 517-20

5. Lenders JW, Eisenhofer G, Mannelli M, Pacak K: Phaeochromocytoma. Lancet, 2005; 366(9486): 665-75

6. Erem C, Kocak M, Onder Ersoz $\mathrm{H}$ et al: Epinephrine secreting cystic pheochromocytoma presenting with an incidental adrenal mass. Endocrine, 2005; 28(2): 225-30 